
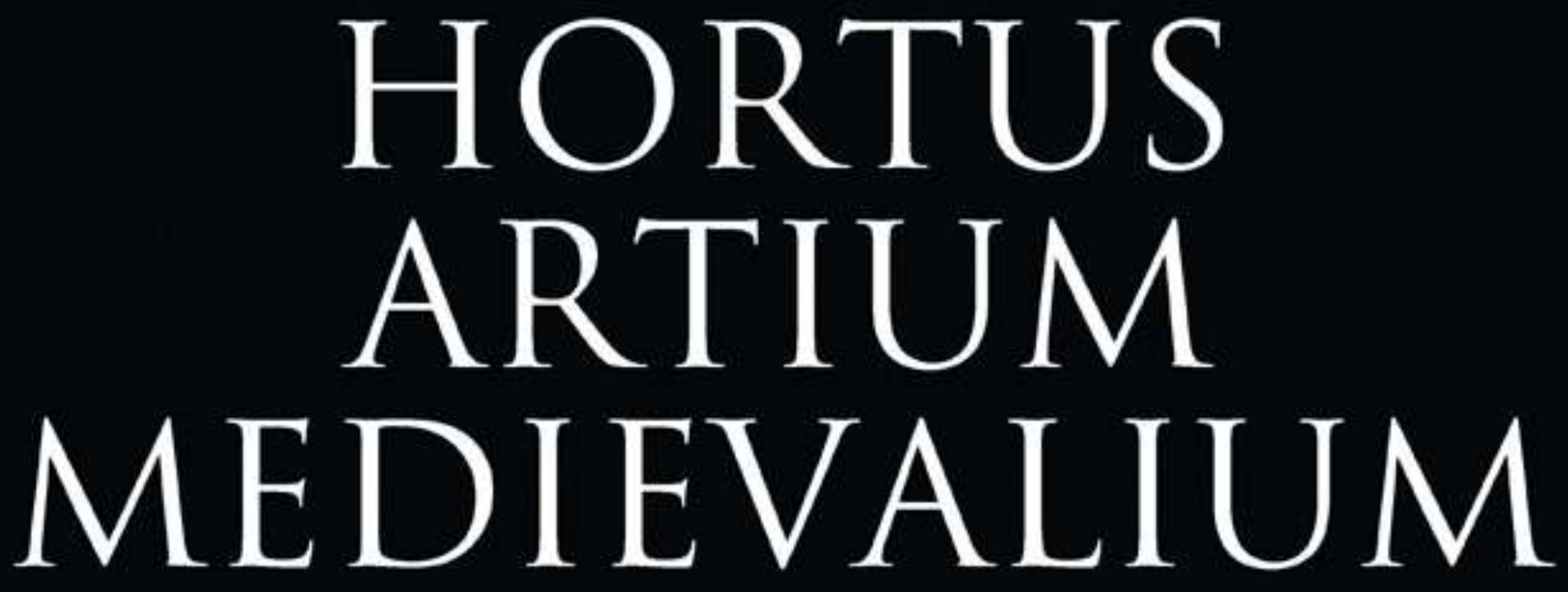

Journal of the International Research Center for Late Antiquity and Middle Ages, Vol. 20/1, Zagreb-Motovun, 2014. 


\section{L'ALBERO DI IESSE NEL XII SECOLO FRA OCCIDENTE E ORIENTE: NOTE SUL PERDUTO MOSAICO DELLA BASILICA DELLA NATIVITÃ A BETLEMME}

SIMONE PIAZZA

UDC: 726.54:75.052(569.4)"11"

Original scientific paper

Manuscript received: 07. 02. 2014.

Revised manuscript accepted: 30. 03. 2014.

DOI: $x x x x x x x x x x x x x x$
S. Piazza

Universite Paul Valery - Montpellier 3 Route de Mende 34199 Montpellier France

The Tree of Jesse, a depiction of Christ's genealogy based on the prophecy of Isaiah (11, 1-2) and on the Gospel of Matthew (1, 1-17), was one of the subjects of the mosaic decoration of the Church of the Nativity in Bethlehem, accomplished in 1169. The work has been entirely destroyed, but it is attested in the counter-façade by Late Medieval and Modern sources. On the basis of this evidence, this paper suggests a reflection both on the iconographic model of the biblical theme, probably of Western origin, and its hypothetical formal features, most likely in line with the Byzantine style of the mosaics which are still visible in the basilica. A tentative reconstruction drawing of the biblical theme completes the analysis.

Keywords: Bethlehem, Church of the Nativity, Tree of Jesse, mosaic, 12th century, Holy Land, Byzantine art, prophets, Sybil

Com'è noto, la basilica della Natività di Betlemme conserva brani significativi del rivestimento musivo risalente all'epoca delle crociate (fig. 1). Ad esso appartiene un'iscrizione bilingue, in latino ${ }^{1}$ e greco ${ }^{2}$, sopravvissuta solo in parte, sul lato meridionale del bema ${ }^{3}$ ma trascritta integralmente fin dall'età medievale ${ }^{4}$. Il testo menziona $\mathrm{i}$ nomi dei personaggi coinvolti nell'impresa e consente quindi di circoscriverne ad annum la datazione: la decorazione venne eseguita nel $1169^{5}$, al tempo del franco Amalrico, quinto re di Gerusalemme (1162-1174), dell'imperatore bizantino Emanuele Comneno (1143-1180), e di Radolfo, vescovo di Betlemme (1155-1174), per mano del mosaicista Efrem, di provenienza siriaca a giudicare dall'origine del nome ${ }^{6}$. Da iscrizioni musive ubicate in altre zone della basilica sappiamo che l'opera fu compiuta anche grazie alla partecipazione del veneziano Zan, forma dialettale di "Johannes", che ha lasciato il suo nome, in lettere greche, presso l'abside meridionale ${ }^{7}$, e di un Basilius pictor, che si firma in latino e in siriaco sul muro nord della navata ${ }^{8}$. Bastano queste iscrizioni per cogliere la cifra multietnica del contesto culturale che ha dato origine ai mosaici betlemitici ${ }^{9}$, frutto dell'alleanza, strategica quanto effimera, tra il re franco-latino e l'imperatore di Bisanzio, entrambi intenzionati a mantenere il controllo su uno dei luoghi di culto più importanti della Terra Santa, contenente al suo interno la venerata grotta della natività di Cristo.

Quanto al contenuto del programma figurativo, brani di mosaico ancora in loco e descrizioni di viaggiatori, a partire dal XIV secolo, consentono di ricostruirne l'assetto d'insieme. E' il francescano Niccolò da Poggibonsi, per primo, intorno alla metà del Trecento, a citare il soggetto del mosaico absidale, la Vergine fra Abramo e David, tema successivamente andato perduto $^{10}$. Un ciclo cristologico era dislocato nella zona presbiteriale, ove rimangono porzioni significative di diverse scene, fra le quali l'Incredulità di San Tommaso, l'Ascensione, la Trasfigurazione e l'Ingresso a Gerusalemme (fig. 2) ${ }^{11}$. La navata centrale, come attestano i lacerti della parete sud e l'ampio brano superstite del muro nord, riprodotto integralmente in un'incisione del Ciampini

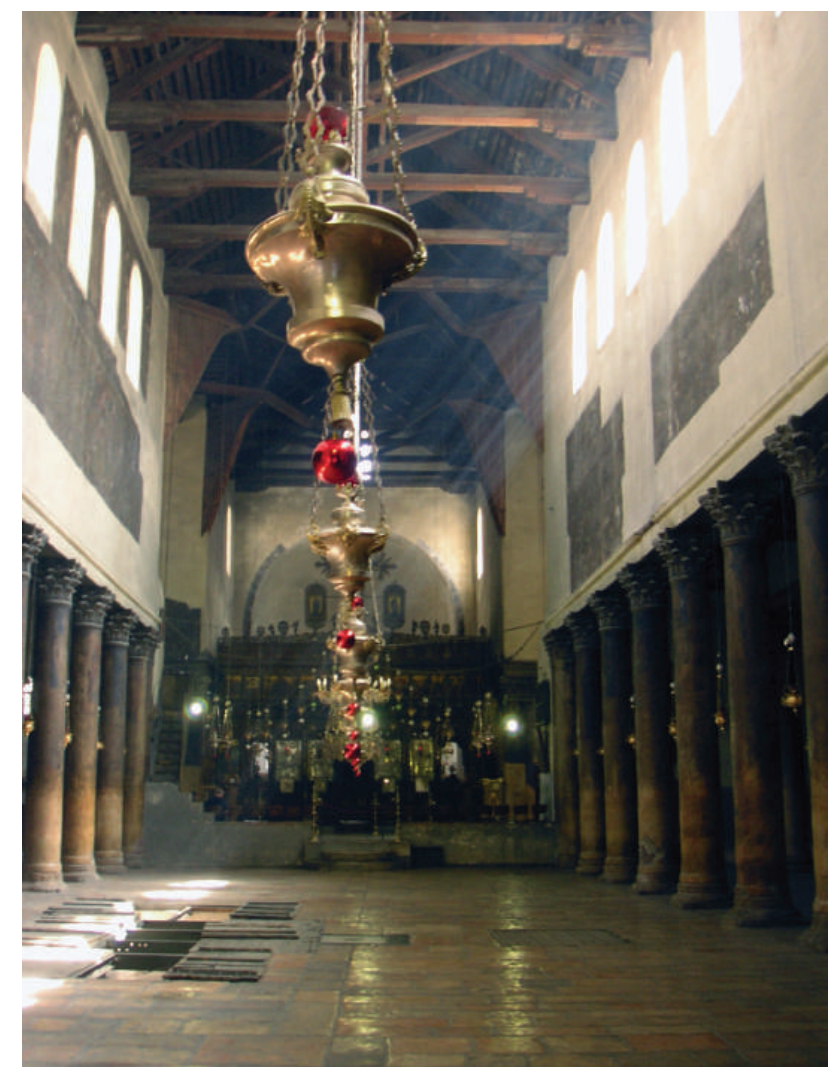

Fig. 1: Betlemme, basilica della Natività, navata centrale con resti della decorazione musiva

(fig. 3) $)^{12}$, ospitava una decorazione continua su tre registri: in alto, tra le finestre, una processione di angeli; nella fascia mediana, una serie di architetture alternata a motivi fitomorfi, con estratti dei concili ecumenici e provinciali della Chiesa d'Oriente, in lingua greca ${ }^{13}$; nel margine inferiore un'interminabile teoria di progenitori di Cristo, tratta dal 


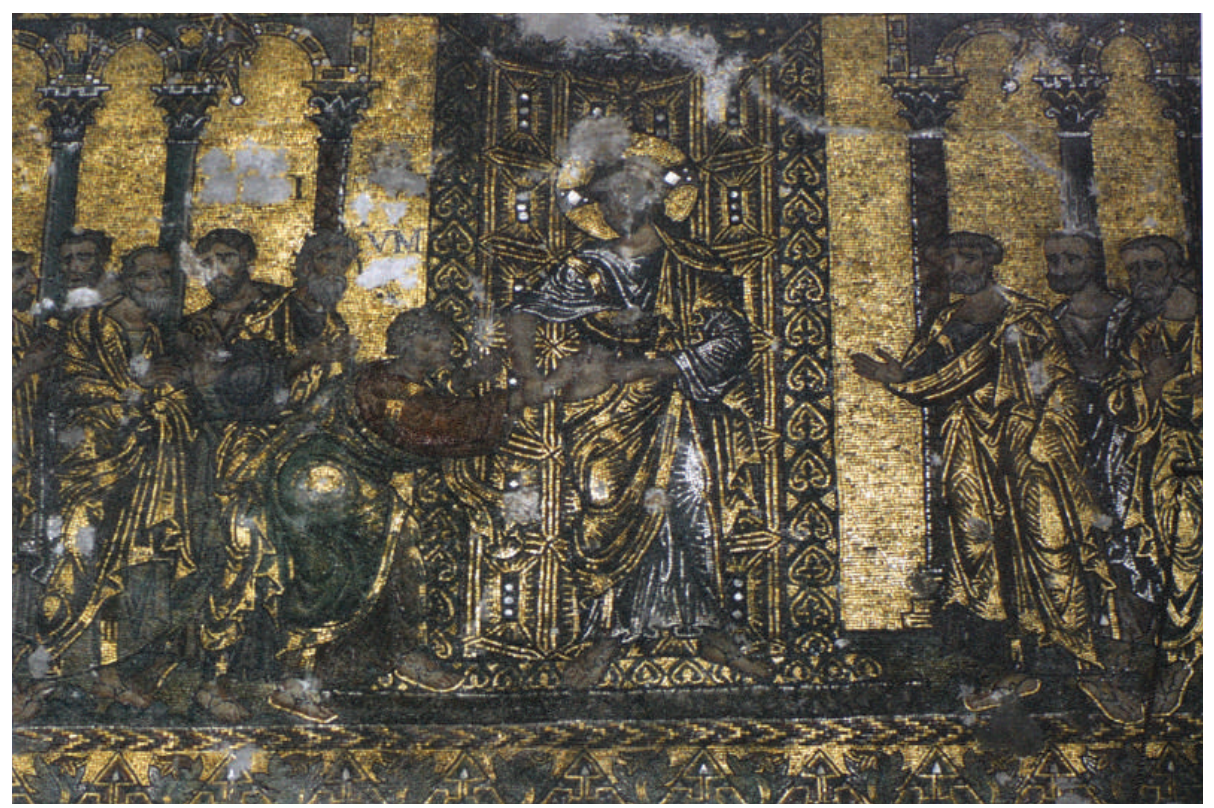

Fig. 2: Betlemme, basilica della Natività, presbiterio, Incredulità di san Tommaso

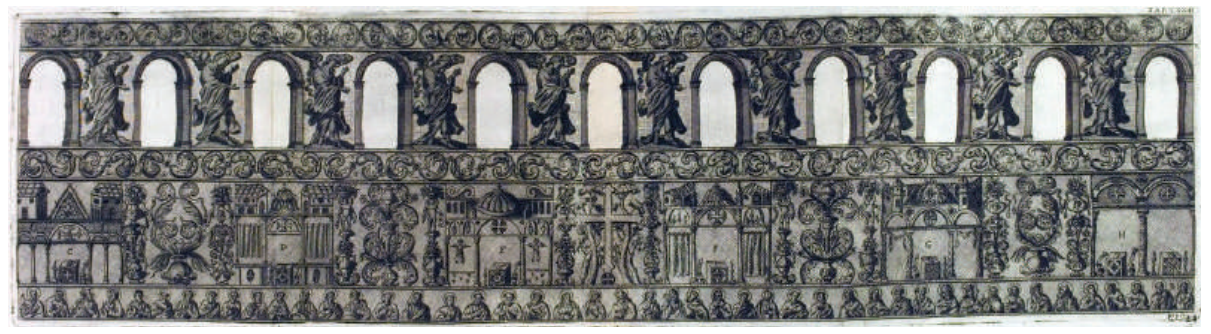

Fig. 3: Betlemme, basilica della Natività, navata centrale, mosaico della parete nord (da Ciampini 1693).

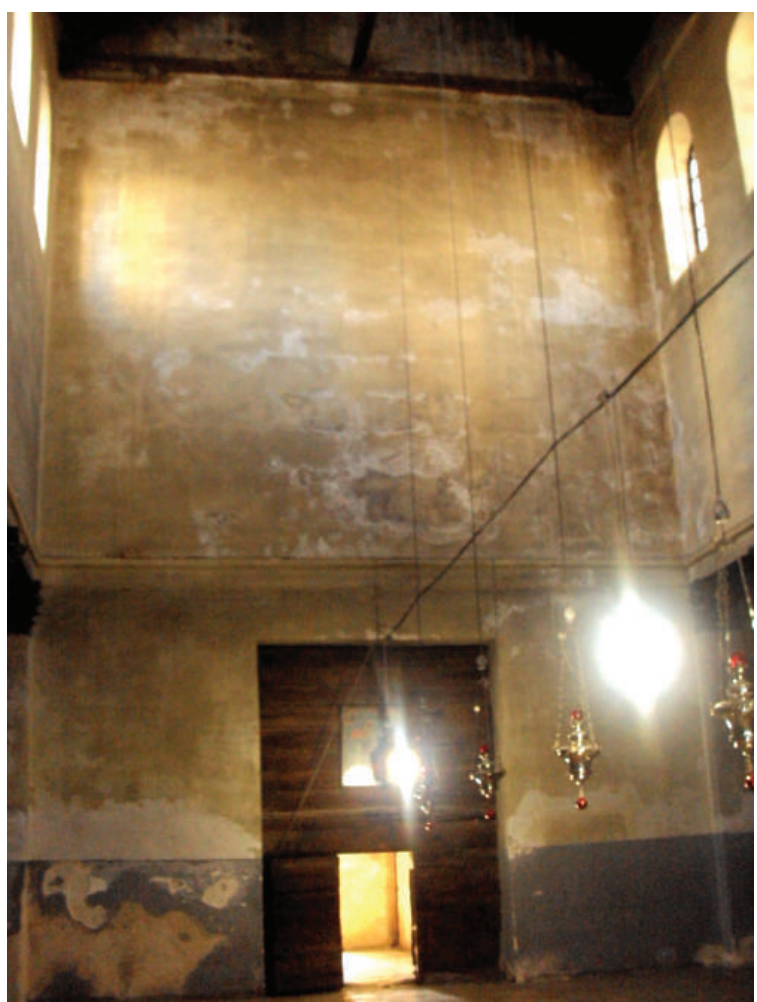

Fig. 4: Betlemme, basilica della Natività, controfacciata vangelo di Matteo lungo la parete meridionale (Mt 1, 1-17) e di Luca in corrispondenza della parete nord (Lc 3, 23-38) ${ }^{14}$. Infine, sulla controfacciata, ove non s'è conservata neppure una tessera di mosaico (fig. 4), sempre Niccolò da Poggibonsi menziona di nuovo gli antenati di Gesù, ma stavolta inseriti all'interno di un organismo arborescente, secondo la versione iconografica dell'Albero di Iesse ${ }^{15}$.

Nell'ambito degli studi dedicati ai mosaici betlemitici, l'attenzione nei confronti di questo tema è passata in secondo piano - evidentemente a causa della perdita totale della memoria fisica dell'opera - rispetto a quella rivolta ad altri soggetti, raffigurazione dei concili in primis ${ }^{16}$. Eppure, l'immagine dell'Albero di Iesse della basilica della Natività desta particolare interesse, in ragione del valore emblematico della sua presenza nel luogo-memoria dell'Avvento di Cristo, per via dello spazio privilegiato che viene ad occupare all'interno della navata, per l'appartenenza al tema della genealogia di Cristo rappresentante il leit-motiv del programma figurativo della basilica, e per altre ragioni che verranno qui di seguito esplicitate.

Onde risalire all'assetto compositivo dell'Albero di Iesse di Betlemme giova rivolgere lo sguardo ad un'opera che costituisce al tempo stesso il precedente iconografico più noto e una delle prime versioni monumentali giunte fino a noi, anche se non la più antica: la vetrata del coro di Saint-Denis, commissionata dall'abate Suger negli anni 1140-1144, ancora oggi ben leggibile nel suo insieme sebbene oggetto di pesanti rifacimenti nel corso dei restauri dell' 800 che ne hanno alterato le singole parti (fig. 5) ${ }^{17}$. 


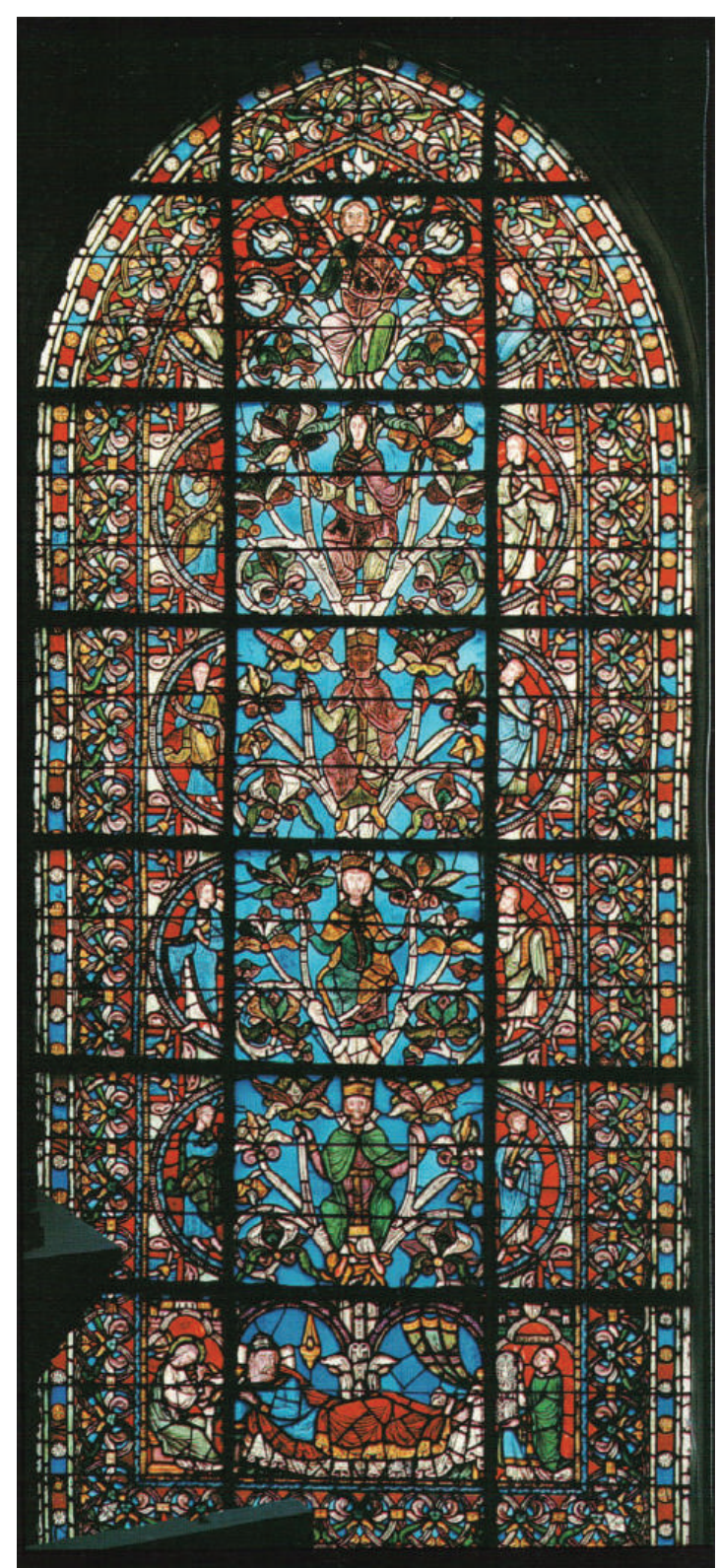

Fig. 5: Abbaziale di Saint-Denis, coro, vetrata dell'Albero di Iesse

Come si evince dall'esempio francese, il tema dell'Albero di Iesse nasce dalla fusione di due fonti bibliche, la profezia veterotestamentaria di Isaia e la genealogia di Cristo evocata nell' incipit del vangelo di Matteo ${ }^{18}$. Il primo versetto del capitolo 11 di Isaia ("Un germoglio spunterà dal tronco di Iesse, un virgulto germoglierà dalle sue radici”) ha dato origine alla figura del vecchio progenitore disteso e addormentato, dal cui fianco nasce il fusto dell'albero. Il passo successivo della medesima fonte biblica, ove viene menzionato sette volte lo "spirito del Signore" 19 , ha ispirato il coronamento della rappresentazione, con sette colombe dello Spirito Santo che convergono verso l'immagine centrale di Cristo. Quest'ultimo, invece, insieme a tutti gli altri personaggi che popolano i rami dell'albero, è frutto dell'interpretazione dei versetti evangelici. Nella genealogia riportata da Matteo, è infatti scritto che "Iesse generò il re David. David il re Salomone" (Matteo, I, 6) e alla fine del lungo elenco degli antenati viene detto che "Giacobbe generò Giuseppe, lo sposo di Maria, dalla quale è nato Gesù chiamato Cristo" (Matteo, I, 16). Secondo un ordine dispositivo che verrà mantenuto nelle versioni successive, lungo il tronco dell'albero di Saint-Denis sono allineati i re biblici, contraddistinti dalle teste coronate ${ }^{20}$. Più in alto è la Vergine, a sua volta sovrastata da Cristo. Ai lati, tra i girali, trovano posto i profeti esibenti cartigli con i loro vaticini.

Quando, sul finire degli anni '60 del XII secolo, il cantiere della basilica di Betlemme realizzò il mosaico sopra l'ingresso, l'Albero di Iesse aveva iniziato a diffondersi in Occidente soltanto da qualche decennio e, verosimilmente, non aveva ancora varcato la soglia del mondo greco-orientale, visto che le prime testimonianze iconografiche bizantine risalgono al XIII secolo ${ }^{21}$. Le iscrizioni contenute nel mosaico della controfacciata della chiesa betlemitica, d'altra parte, erano in lingua latina, come si deduce dalla descrizione lasciataci da Francesco Quaresmi nel suo trattato sulla Terra Santa $(1639)^{22}$. Compiendo la lettura dal basso verso l'alto, l'erudito francescano riconosce dapprima Gioele, di cui riporta il verso 18 del capitolo 3 ("In quel giorno le montagne stilleranno vino nuovo e latte scorrerà per le colline"). Poi, di seguito, Amos 8, 9 ("In quel giorno farò tramontare il sole a mezzodì e oscurerò la terra in pieno giorno") e Naum, 1 , 19 ("Ecco sui monti i passi d'un messaggero, che annuncia pace”). Distingue, inoltre, il nome di "Ezechiele" e accanto trascrive le lettere associate ad un altro profeta senza però arrivare all'identificazione ("IHEASPPhA"), come invece è stato in grado di fare, nel secolo scorso, padre Sabino De Sandoli, che ha letto I[SA](HE)AS P[RO]PH[ET] ${ }^{23}$. Al disopra Quaresmi scorge la famosa profezia di Balaam contenuta nel libro dei Numeri ("Una stella spunta da Giacobbe e uno scettro sorge da Israele", Num. 24, 17). Un altro cartiglio era retto da Michea con la profezia del suo quinto capitolo ("Da te, Betlemme di Efrata, uscirà colui che deve essere il dominatore in Israele. E la sua uscita è dall'inizio, dai Giorni dell'eternità", Mich. 5, 2). L'ultima iscrizione ad essere trascritta non appartiene ad un profeta delle sacre scritture bensì ad una Sibilla: "Verrà dal cielo Colui che sarà re per sempre" è infatti un verso contenuto negli Oracula Sybillina, di cui si darà conto a breve. Oltre alle otto figure segnalate dal religioso, ve ne dovevano essere certamente altre: sopra Iesse disteso - ignorato da Quaresmi, diversamente dal suo predecessore Niccolò di Poggibonsi, che lo scambia per Abramo ${ }^{24}$-, non potevano non esserci, lungo il fusto verticale dell'albero, i re biblici, la Vergine e Gesù Cristo. I profeti, poi, a giudicare dall'ampiezza della superficie della controfacciata, assai verosimilmente erano più numerosi. L'incompletezza dell'analisi del Quaresmi è dovuta, con ogni probabilità, allo stato lacunoso in cui versava il mosaico già ai suoi tempi ${ }^{25}$.

Alcuni paralleli con i profeti identificati dal Quaresmi s'incontrano nelle precedenti versioni delle vetrate di Saint-Denis e di Chartres. Nell'opera di Suger ritroviamo, oltre ad Isaia, anche Gioele e Amos, i cui filatteri presentano testi che corrispondono a quelli della basilica della Terra Santa $^{26}$. A Chartres, invece, dove i progenitori sono di numero maggiore e ciascuno di essi è riconoscibile soltanto grazie all'iscrizione onomastica perché i rotoli sono privi di versetti biblici, riscontriamo la presenza di Ezechiele, Balaam, Michea e $\mathrm{Naum}^{27}$. Al di là di qualche sporadica coincidenza, tuttavia, nei contesti citati la disposizione dei profeti non risulta sovrapponibile. E d'altra parte, il confronto fra un nutrito numero di testimonianze dell'Albero di Iesse, risalenti ai secoli XII e XIII, ha consentito di appurare che l'ordine distributivo dei profeti varia da caso a caso ${ }^{28}$. 


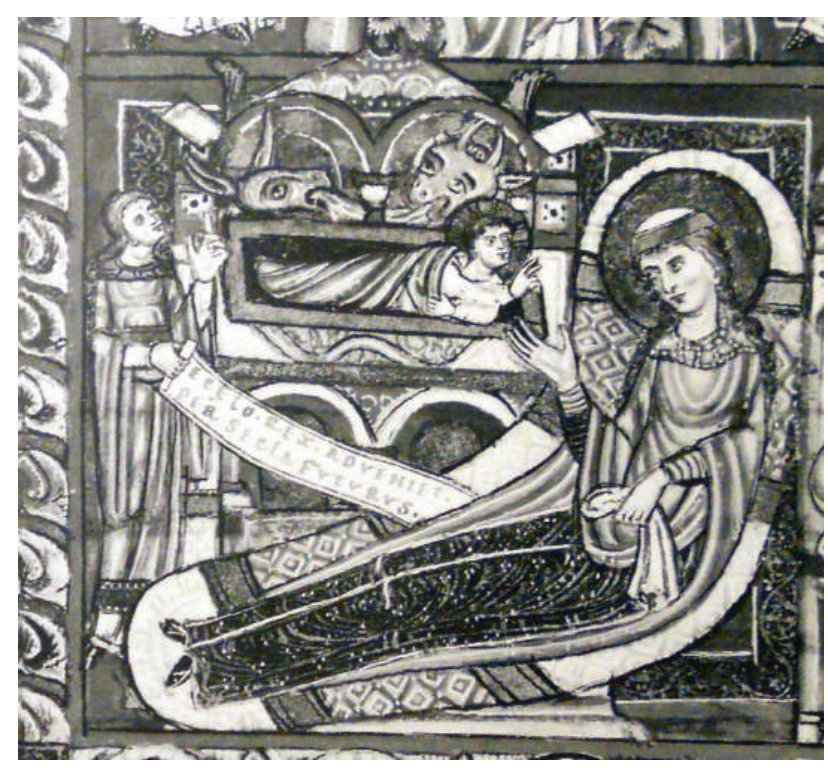

Fig. 6: Cleveland, Museum of Arts, ms. 33.445, part. della Natività con la Sibilla annunciante la profezia

Nell'ambito delle prime versioni iconografiche dell'albero degli antenati, la variabilità nella scelta dei personaggi secondari emerge in tutta la sua evidenza se si prende in considerazione la presenza, a Betlemme e non a Saint-Denis né a Chartres, della Sibilla, prophetissa di origine pagana, associata ai vaticini biblici dell'Avvento nei testi patristici e liturgici a partire dai primi secoli del Cristianesimo ${ }^{29}$. Essa si trova all'interno della rappresentazione dell'Albero di Iesse fin dalle origini, anche se i casi noti sono piuttosto rari: compare infatti per la prima volta nella formella di bronzo della porta di Verona ${ }^{30}$; poi, sul finire del XII secolo, nel codice miniato della Biblioteca Municipale di Douai (Ms. 340, fol. 11r) ${ }^{31}$, e di nuovo, più o meno nello stesso periodo, nel salterio della regina Ingeborg di Danimarca al Musée Condé di Chantilly ${ }^{32}$. In questo caso la Sibilla esibisce un'iscrizione nel cartiglio (Omnia cessabunt, tellus confracta peribit, “Tutto cesserà, la terra finirà frantumata"), che non coincide con quella attestata a Betlemme ma proviene dalla medesima fonte, il cosiddetto "Acrostico della Sibilla Eritrea", scritto oracolare composto con le iniziali di ventisette versi in esametri greci che in verticale formano la frase ("Gesù Cristo figlio di Dio Salvatore") ${ }^{33}$. Già nel II secolo d. C. l'acrostico si trova nei citati Oracula Sibyllina, un centone di quattordici libri che raccoglie i vaticini delle Sibille, redatto forse ad Alessandria d'Egitto ${ }^{34}$. I versi vengono riportati, in latino, nel De Civitate Dei di Agostino e numerosi sono gli autori cristiani ad aggiungerli alle profezie bibliche, come segno della prefigurazione dell'Avvento di Cristo nel mondo pagano ${ }^{35}$. L'iscrizione della sibilla raffigurata sul Salterio di Chantilly corrisponde alla ventunesima riga dell'acrostico ("tutto cesserà, la terra finirà frantumata") ${ }^{36}$, quella del mosaico di Betlemme alla seconda ("verrà dal cielo Colui che sarà re per sempre") ${ }^{37}$. Il medesimo verso si ritrova anche nella scena della Natività della pagina di un codice sassone, dell'ultimo quarto del XII secolo, conservata al Museum of Arts di Cleveland (ms. 33.445), con chiaro riferimento alla profezia della nascita di Cristo derivante dal mondo pagano (fig. 6) ${ }^{38}$. Diversamente, nella decorazione pittorica di Sant'Angelo in Formis, realizzata al tempo dall'abate Desiderio (1072-1087), l'iscrizione che la Sibilla esibisce nel suo filatterio coincide con il primo verso dell'acrostico, in-

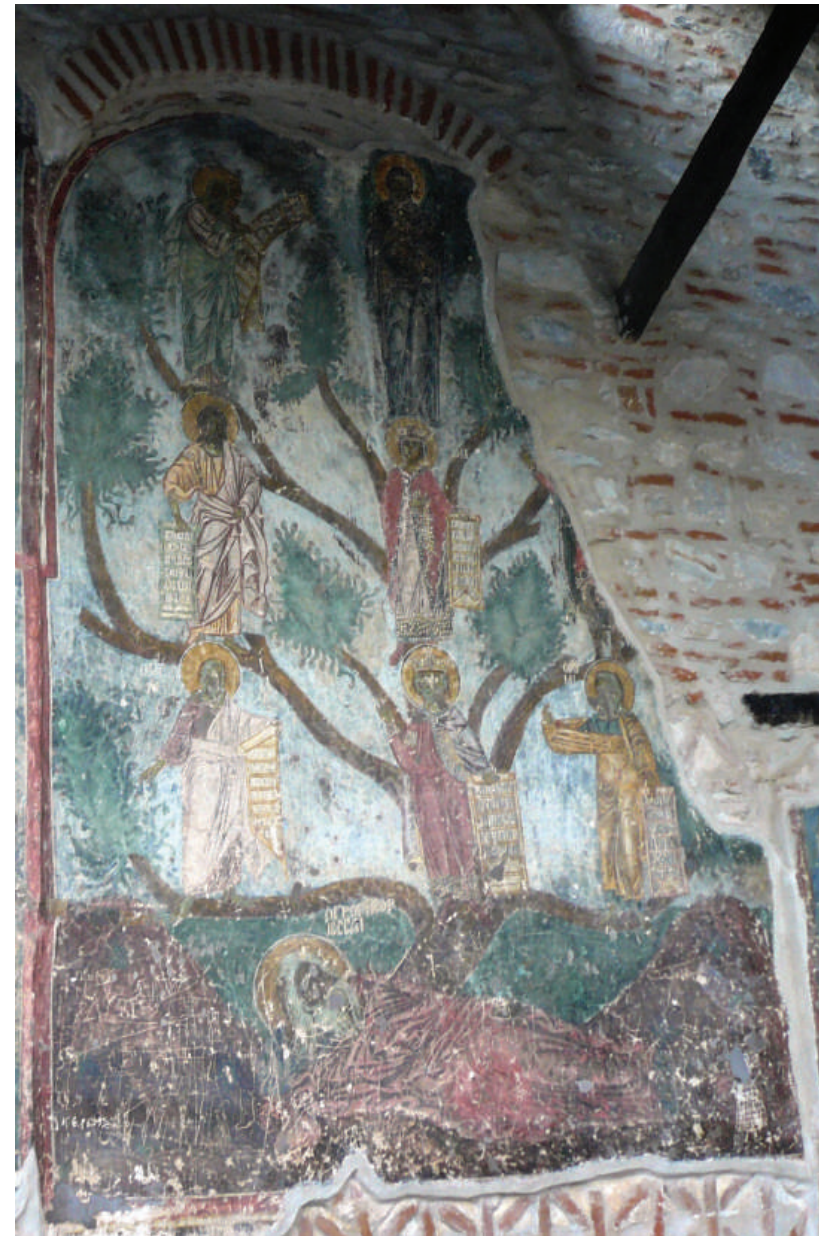

Fig. 7: Kastoria, chiesa della Mavriotissa, Albero di Iesse

terpretato come profezia del Giudizio universale: "Segno di giudizio la terra sarà madida di sudore" ${ }^{39}$. Sebbene l'iconografia dell'Albero di Iesse non fosse ancora in circolazione all'epoca dell'esecuzione dei dipinti santangiolesi, la Sibilla, i profeti e i re biblici, sopra le colonne della navata centrale della chiesa campana, rappresentano i cardini dell'intero programma decorativo, illustrante la storia della salvezza del genere umano, dalla Genesi al Giudizio.

Gli esempi citati aiutano a capire il significato della presenza della Sibilla tra i profeti betlemitici, che acquista ancor più concretezza se si prende in considerazione un breviario del Santo Sepolcro conservato al museo Condé di Chantilly, databile al XIII secolo, ma in realtà copia di una versione più antica, probabilmente risalente agli anni '70 del XII secolo ${ }^{40}$ e quindi verosimilmente contemporanea ai mosaici della basilica della Natività. Il manoscritto contiene numerose rubriche sulla liturgia del patriarcato latino di Gerusalemme. Nella lectio VI dell'officio di Natale veniva pronunciata la frase: "Comunichiamo a tutti anche ciò che la Sibilla ha proclamato del Cristo”, seguita dall'acrostico, del quale il manoscritto riporta i primi e gli ultimi esametri ${ }^{41}$. Com'è spiegato nel breviario, la notte della vigilia dell'Avvento questi versi, insieme a quelli della genealogia di Cristo tratti dal vangelo di Matteo, venivano cantati all'interno della basilica betlemitica in presenza del patriarca di Gerusalemme ${ }^{42}$. L'indomani mattina, in processione, il clero intonava l'antifona "O Maria Iesse Virga" ${ }^{43}$. L'Albero di Iesse della controfacciata della basilica di Betlemme, con la sua 


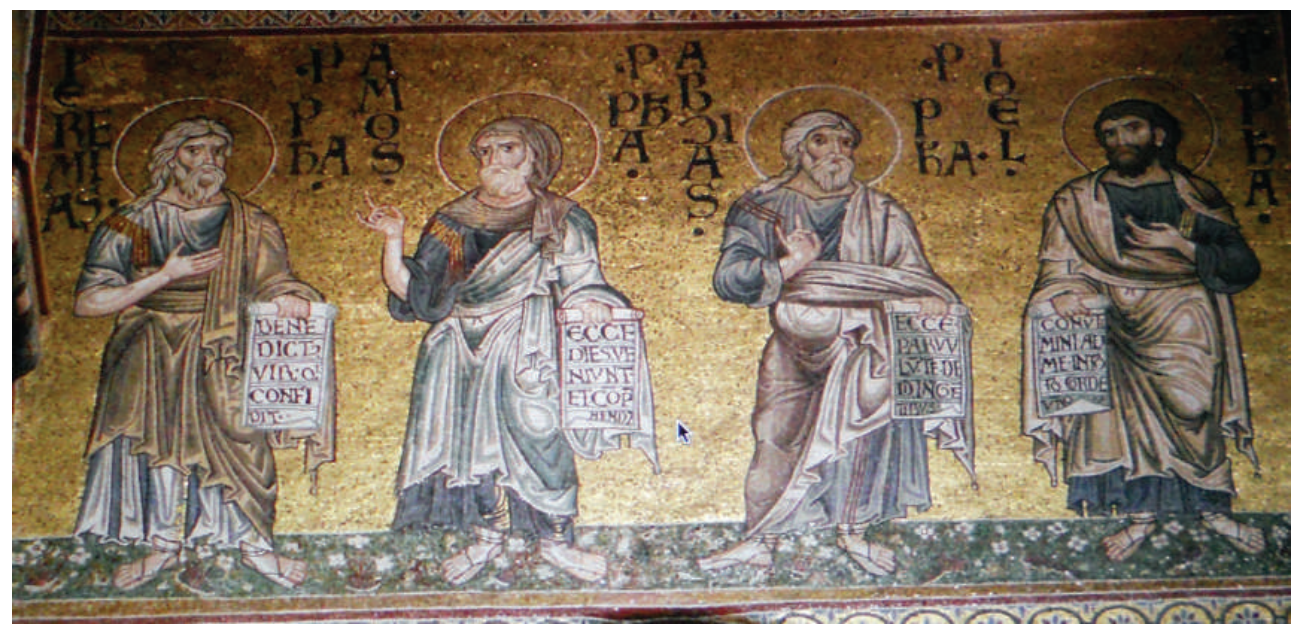

Fig. 9: Basilica di Monreale, coro, part. del mosaico con i profeti Geremia, Amos, Abdia e Gioele

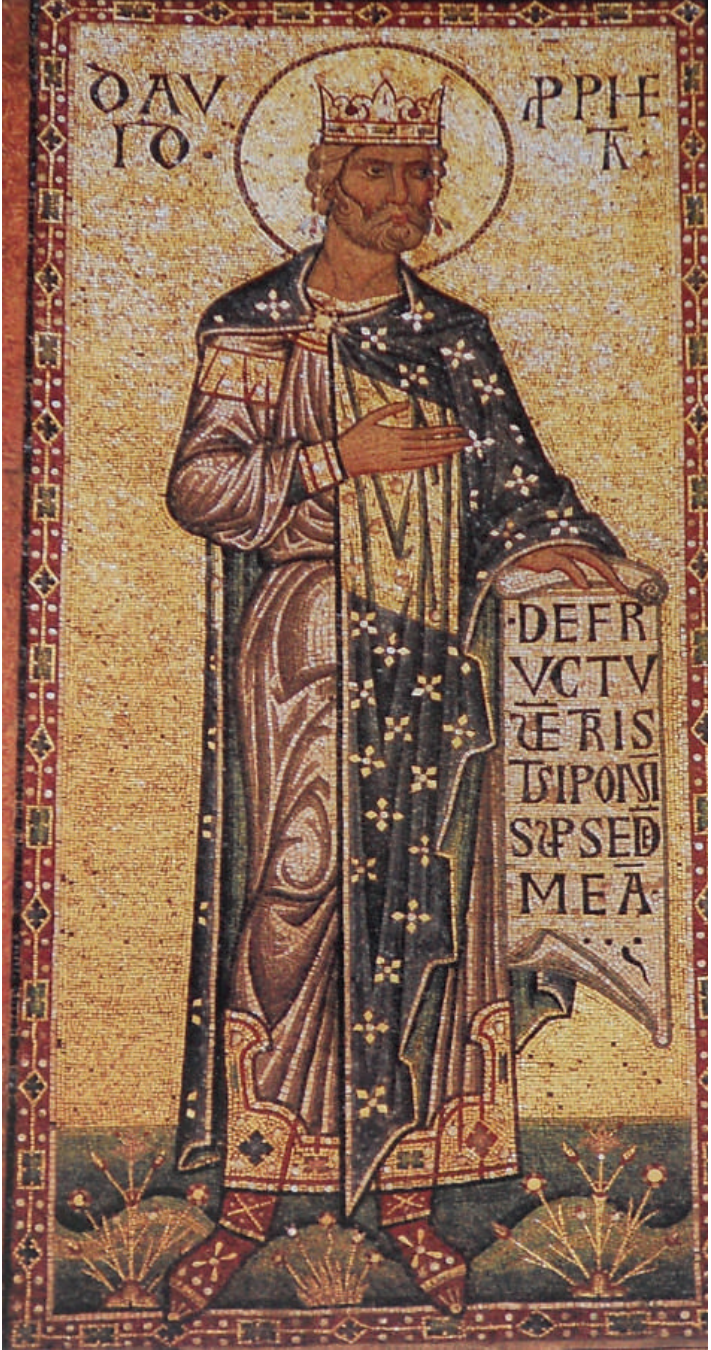

Fig. 8: Venezia, San Marco, pannello musivo della navata raffigurante il re David

Sibilla, trova, pertanto, piena giustificazione nel coevo rito latino di Terra Santa.

Se, come abbiamo visto, la matrice iconografica dell'Albero di Iesse proveniva dalla cultura occidentale, è assai probabile che, per quanto attiene agli aspetti formali, l'opera si adeguasse ad un linguaggio di matrice greca. I superstiti pannelli musivi con le scene cristologiche ricalcano, infatti, un ductus stilistico d'impronta bizantina ${ }^{44}$, pur mediato, verosimilmente, dalla mano di artisti locali e veneziani $^{45}$ (fig. 2). A supporto di tale ipotesi giova ricordare un dettaglio che emerge dall'opera del Quaresmi, a nostro avviso non trascurabile: le iscrizioni dei profeti, che l'autore ricopia meticolosamente, nell'intento di imitare i caratteri paleografici impiegati dai mosaicisti, hanno andamento verticale o orizzontale, segno che i cartigli musivi presentavano, presumibilmente, forma più o meno rettangolare. Tant'è vero che il Quaresmi li definisce "tabelle" o "schedule" ${ }^{46}$. Diversamente, nelle vetrate francesi i filatteri degli antenati assumono l'aspetto di lunghi nastri ondivaghi (fig. 5). Il profilo rettangolare è invece attestato in ambito greco, all'interno dell'Albero di Iesse dipinto all'ingresso della Mavriotissa di Kastoria (XIII secolo ${ }^{47}$, ove i profeti e re biblici, tanto nella foggia che nella posa, ricalcano modelli inveterati, di norma riscontrabili all'interno delle chiese mediobizantine lungo la superficie interna del tamburo della cupola centrale (fig. 7$)^{48}$.

Per trovare referenti formali più vicini al caso di Betlemme conviene rivolgere lo sguardo ai due grandi centri artistici del Mediterraneo in attività negli stessi anni, i cantieri del duomo di Monreale e della basilica di San Marco a Venezia, che condividono con la decorazione betlemitica il medium pittorico e il linguaggio stilistico bizantino, nonostante gli apporti locali. I profeti del coro del duomo monrealese (fig. 8) ${ }^{49}$ e quelli, successivi di qualche decennio, sulle pareti della navata della chiesa marciana (fig. 9) ${ }^{50}$, possono dare un'idea dell'aspetto assunto, verosimilmente, dagli omologhi personaggi sulla controfacciata dell'edificio di Betlemme, di cui proponiamo una restituzione grafica, a titolo puramente esemplificativo (fig. 10) ${ }^{51}$.

All'interno della sterminata compagine musiva dell'edificio monrealese si registra l'assenza dell'iconografia dell'Albero di Iesse e al tempo stesso la presenza di otto busti clipeati di profeti fra racemi fogliati, proprio intorno al gigantesco Pantocrator dell'abside, ai lati del Cristo Emmanuel, allusione alle profezie di Isaia (Isaia, 7,14 e 8,8) ${ }^{52}$. E d'altra parte, per misurare l'importanza conferita agli antenati di Cristo all'interno del duomo siciliano occorre tener conto anche dei ventisei medaglioni dei sottarchi all'incrocio col transetto - tra cui figura pure Iesse -, oltre ai dodici del coro già menzionati ${ }^{53}$. Nel XII secolo inoltra- 

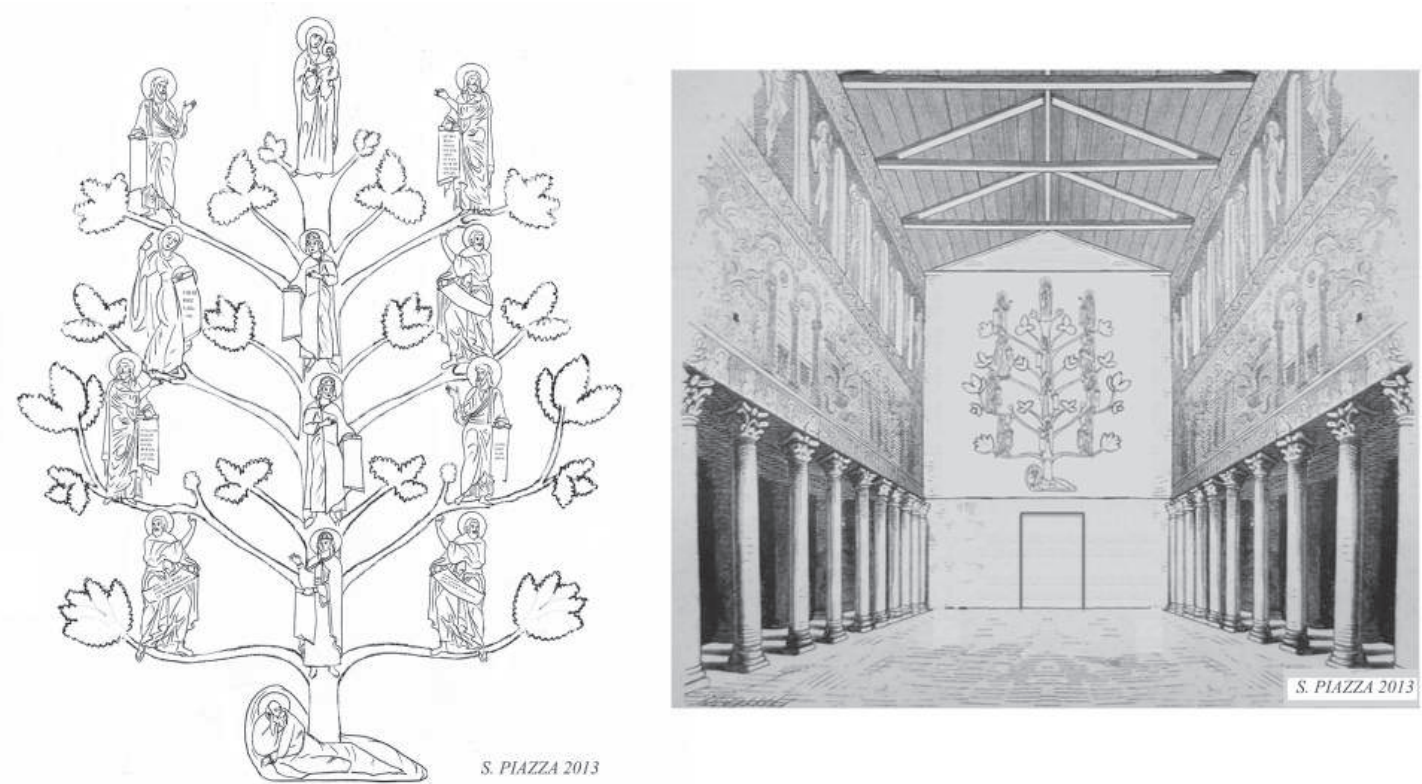

Fig. 10: Betlemme, basilica della Natività, ipotesi di restituzione grafica dell'Albero di Iesse nel contesto della decorazione musiva della navata (Piazza 2013)

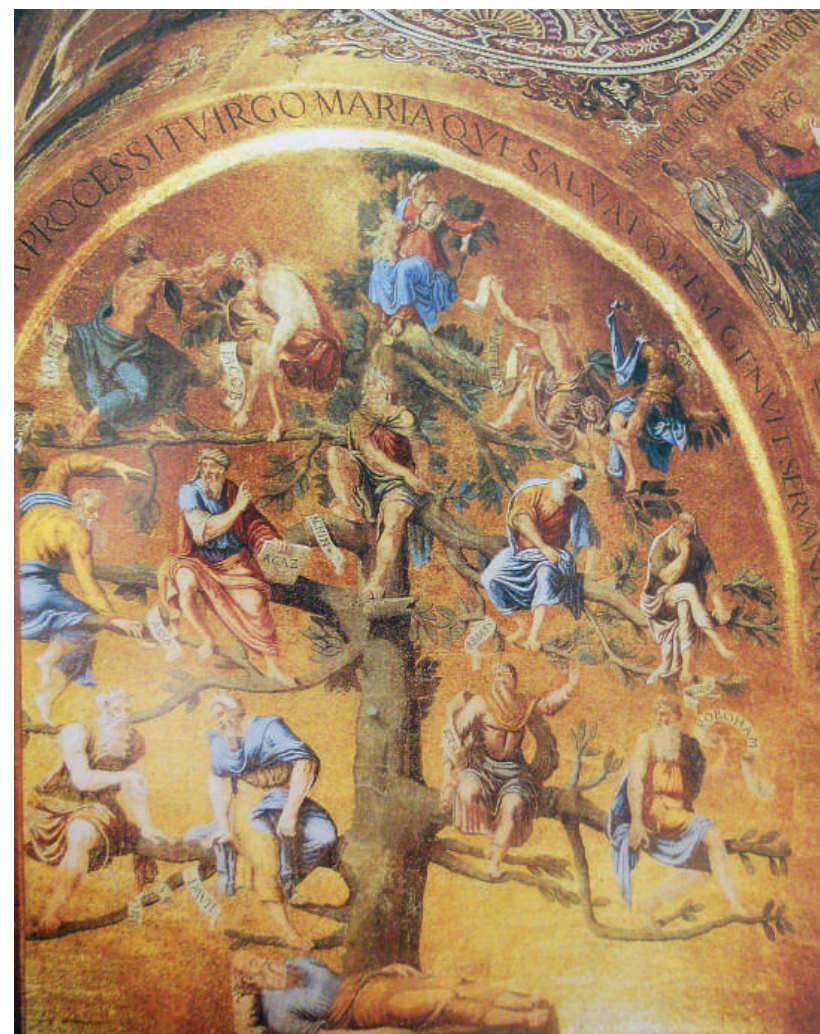

Fig. 11: Venezia, San Marco, parete di fondo del transetto nord, l'Albero di Iesse di Domenico Bianchini (metà XVI sec.)

to, insomma, nell'ambito dei programmi figurativi delle basiliche di committenza aulica, la scelta di rappresentare l'Albero di Iesse non costituisce l'unica soluzione possibile atta a porre l'accento sulle profezie bibliche dell'Avvento. A ragione, quindi, Louis Réau accostava l'albero della genealogia di Cristo alle statues-colonnes delle facciate del gotico francese, raffiguranti non i monarchi d'oltralpe - come teneva a precisare lo studioso sfatando un antico mito storiografico - bensì i profeti e i re del Vecchio Testamento ${ }^{54}$.

Un caso diverso è rappresentato dalla basilica marciana, dal momento che a Venezia esiste un vero e proprio Albero di Iesse, occupante l'intera e vastissima superficie musiva della parete di fondo del transetto nord ${ }^{55}$. Si tratta, però, di un mosaico tardo rinascimentale, opera attribuita all'artista Domenico Bianchini, databile alla metà del '500 (fig. $11)^{56}$. Otto Demus considerava il pannello musivo frutto di un intervento concepito ex novo in età moderna e non il rifacimento di una versione medievale danneggiata dal tempo ${ }^{57}$, tesi ribadita anche di recente, in virtù delle considerevoli dimensioni della superficie musiva, che sono sembrate spropositate per un'opera concepita nel Medio $\mathrm{Evo}^{58}$. A ben riflettere, invece, proprio il gigantismo appare come elemento a favore di una derivazione medievale dell'albero veneziano, se si tiene conto delle proporzioni monumentali del mosaico di Betlemme, delle vetrate di Saint-Denis e di Chartres, dei dipinti murali di Ronciglione e di Kastoria, per non ricordare che gli esempi più antichi ${ }^{59}$. Le citate versioni medievali, oltretutto, condividono con il pannello marciano la cornice curvilinea dell'estremità superiore, con la sola eccezione del caso betlemitico di cui ignoriamo l'originario profilo. C'è poi da dire che in Occidente l'Albero di Iesse, dopo aver conosciuto un grande successo in età gotica, a partire dal Quattrocento si fa via via sempre più raro. Occorrerà quindi riprendere in altra sede la questione di una possibile origine medievale della versione marciana, che merita di essere approfondita perché lascia intravedere l'eventualità di uno scambio di modelli fra Venezia e Betlemme. 
1 “Il re Amalrico, custode della virtù, amico liberale, compagno dell'onestà, nemico dell'empietà, amante della giustizia, cultore della pietà, vendicatore del crime, regnava come quinto re e comandava ai Greci Emmanuele, donatore generoso e pio imperatore; viveva qui Presule e reggeva la chiesa il degno di onore e benigno Pontefice Radolfo; quando la mano di Efrem, si dice, fece per essi la soave (pittura)": B. BAGATTI, Gli antichi edifici sacri di Betlemme in seguito agli scavi e restauri praticati dalla Custodia di Terra Santa (1948-51), Gerusalemme, 1952, p. 60.

2 "La presente opera è stata finita per mano di Efrem, pittore e musaicista, sotto il regno del grande Imperatore Emmanuele Porfirogenete Comneno, ai giorni del gran re di Gerusalemme il signor Amalrico, e del santissimo vescovo della santa città di Betlemme, Radolfo, nell'anno 6677, indizione II” (1165), o "nell'anno 6673, indizione XII" (1169): B. BAGATTI, Gli antichi edifici... op. cit., p. 61. Tradizionalemnte la datazione contenuta nell'iscrizione greca, non ben leggibile a causa della lacunosità del mosaico, veniva fatta coincidere con il 1165 (anno 6677 del calendario bizantino, indizione II) o con il 1169 (anno 6673, indizione XII): H. VINCENT, F.-M. ABEL, Bethléem. Le sanctuaire de la Nativité, Paris, 1914, p. 158. Antony Cutler ha però sciolto ogni dubbio in proposito, grazie alla scoperta della trascrizione del testo musivo betlemitico in un foglio di un manoscritto di Gerusalemme risalente al 1182 (Gr. Patr. cod. 57), che riporta la data del 1169: A. CUTLER, Ephraim, mosaicist of Bethlehem: the evidence from Jerusalem, in Jewish Art, 12-13, 1986-87, pp. 179-181 (p. 179).

${ }^{3}$ L'iscrizione latina (cfr. supra nota 1) si trovava lungo la parete meridionale del coro, sulla sinistra, sotto la Dormitio Virginis (B. BAGATTI, Gli antichi edifici... op. cit., p. 60); accanto, sulla destra, in corrispondenza della Presentazione al Tempio, correva l'iscrizione greca (Ibidem, pp. 60-61). Cfr. J. FOLDA, Crusader art. The art of the crusaders in the Holy Land, 1099-1291, Aldershot, 2008, pp. 347-350, fig. 9.8b e tav. 30 a colori. Per una visualizzazione delle due scene nel bema della basilica betlemitica, cfr. G. KÜHNEL, Das Ausschmuckungsprogramm der Geburtsbasilika in Bethlehem. Byzanz und Abendland im Konigreich Jerusalem, in Boreas, 10, 1987, pp. 133-49.

${ }^{4}$ Cfr. H. VINCENT, F.-M. ABEL, Bethléem... op. cit., p. 158.

${ }^{5}$ Cfr. supra, nota 2.

${ }^{6}$ A. CUTLER, Ephraim... op. cit., pp. 179-181.

${ }^{7}$ L. A. HUNT, Art and colonialism: the mosaics of the church of the Nativity in Bethlehem (1169) and the problem of 'Crusader art', in Dumbarton Oaks Papers, 45, 1991, pp. 69-85 (p. 74).

${ }^{8}$ Ibidem.

${ }^{9}$ Ibidem, p. 82; G. KÜHNEL, Twelfth-century decoration of the Church of the Nativity: eastern and western concord, in Ancient churches revealed, Y. TSAFRIR (a cura di), Washington, 1993, pp. 197-203; J. FOLDA, The Art... op. cit., pp. 357; M. ANDALORO, Da Bisanzio al Mediterraneo, in Il Mediterraneo e l'arte nel Medioevo, R. CASSANELLI (a cura di), Milano, 2000, pp. 195-217 (p. 206).

10 “Nella quale (abside) - scrive - c'è lavorato d'opera mosaica la Vergine Maria, e dall'una parte Abraam, e dall'altra David”: N. DA POGGIBONSI, Libro d'Oltramare, Bologna, 1881 (rist. anast. Bologna, 1968), p. 220.

${ }^{11}$ G. KÜHNEL, Das Ausschmuckungsprogramm... op. cit., pp. 138-139, figg. 2-4; L. A. HUNT, Art and colonialism... op. cit., p. 78; J. FOLDA, The Art... op. cit., pp. 357-358.

${ }^{12}$ G. G. CIAMPINI, De sacris aedificiis a Costantino Magno constructis: synopsis historica, Roma, 1693, pp. 150-161, tav. XXXIII tra p. 150 e p. 151.

${ }^{13}$ Ad eccezione del concilio niceno II, di cui è riportato il testo in latino.

${ }^{14}$ G. KÜHNEL, Das Ausschmuckungsprogramm... op. cit., pp. 140-142, figg. 3-4; L. A. HUNT, Art and colonialism... op. cit., pp. 78-81; J. FOLDA, The Art... op. cit., pp. 358-360.

15 “Sopra la grande porta (...) si è figurato l'albero, come nasce dal lato d'Abraam (...), nel primo ramo si è Isaac, nel secondo si è Iacob, e così l'altre ramora tutti i Profeti che profetezarono Iesù Cristo, tutti, ciascuno colla sua profezia in mano": N. DA POGGIBONSI, Libro d'Oltramare... op. cit., p. 219. ${ }^{16}$ G. KÜHNEL, Die Konzilsdarstellungen in der Geburtskirche in Bethlehem: ihre kunsthistorische Tradition und ihr kirchenpolitisch-historischer Hintergrund, in Byzantinische Zeitschrift, 86-87, 1993-1994, pp. 86-107; M. R. MENNA, Immagini e scritture nei mosaici della chiesa della Natività a Betlemme, in Il cammino di Gerusalemme, Atti del II Convegno internazionale di studio (Bari, Brindisi, Trani, 18-22 maggio 1999), M. S. CALÒ MARIANI (a cura di), Bari, 2002, pp. 647-658; M. ANDALORO, Da Bisanzio... op. cit., pp. 198-204.

${ }^{17}$ L. GRODECKI, Les vitraux de Saint-Denis, in Idem, Etudes sur les vitraux de Suger à Saint-Denis, I (Corpus vitrearum, France, Etudes I), Paris, 1976, pp. 71-80; C. GRODECKI, Les fenêtres de l'Incarnation: l'Enfance du Christ, in L. GRODECKI, Etudes sur les vitraux de Suger à Saint-Denis, II (Corpus vitrearum, France, Etudes III), Paris, 1995, pp. 23-28, spec. pp. 23-24; S. PIAZZA, Genesi e fortuna dell'Albero di Iesse nel XII secolo: Sant'Eusebio prima di Saint-Denis, in Le plaisir de l'art du Moyen Âge. Commande, production et réception de l'oeuvre d'art. Mélanges d'histoire de l'art offerts en hommage à Xavier Barral I Altet, Paris, 2012, pp. 819-825.

${ }^{18}$ Per uno sguardo d'insieme sul tema dell'Albero di Iesse nell'arte medievale si veda: A. WATSON, The Early Iconography of the Tree of Jesse, London, 1934; C. LAPOSTOLLE, Albero di Iesse, in Enciclopedia dell'Arte Medievale, I, 1991, pp. 308-13 (con ricca bibliografia).

19 "Su di lui si poserà lo spirito del Signore, spirito di sapienza e di intelligenza, spirito di consiglio e di fortezza, spirito di conoscenza e di timore del Signore", Isaia, 11, 2.

${ }^{20}$ Lungo il tronco dell'albero della vetrata di Saint-Denis si trovano, dal basso verso l'alto, i re David, Salomone e Roboamo, rispettivamente figlio, nipote e bisnipote di Iesse. In versioni iconografiche più ampie e articolate, sopra Roboamo possiamo trovare Abìa e altre generazioni di monarchi biblici, sempre secondo l'ordine genealogico della discendenza.

${ }^{21}$ V. MILANOVIĆ, The Tree of Jesse in the Byzantine mural painting of the thirteenth and fourteenth centuries, in Zograf, 20, 1989, pp. 48-60, spec. p. 40 e bibl. n. 1; S. PIAZZA, Genesi e fortuna... op. cit., nota 12, pp. 823-824.

${ }^{22}$ F. QUARESMIUS, Historica, theologica et moralis Terrae Sanctae elucidatio, Anversa, 1639, vol. II, p. 645.

${ }^{23}$ S. DE SANDOLI, Corpus inscriptionum Crucesignatorum Terrae Sanctae, 1099-1291: testo, traduzione e annotazioni, Jerusalem, 1974, p. 209.

${ }^{24}$ N. DA POGGIBONSI, Libro d'Oltramare... op. cit., p. 219.

${ }^{25}$ Il canonico Alcarotti, sul finire del XVI secolo, lamentava atti vandalici inferti ai mosaici della basilica betlemitica: i "Turchi tirano alle figure delle archibugiate dove non arrivano in altro modo a distruggerle”: G. F. ALCAROTTI, Del Viaggio di Terra Santa, Novara, 1596, p. 149; B. BAGATTI, Gli antichi edifici... op. cit., p. 59. Lo stesso Quaresmi fa riferimento allo stato d'incuria delle superfici musive: "Sacratissima haec Ecclesia olim pulcherrimis picturis et scripturis exornabatur, quas pro maiori parte edax voravit tempus, et infidelium barbarie set iniuria compsumpsit”, F. QUARESMIUS, Historica... op. cit. p. 645.

${ }^{26}$ L. GRODECKI, Les vitraux... op. cit., pp. 76-77.

${ }^{27}$ C. MANHES-DEREMBLE, Les vitraux narratifs de la cathédrale de Chartres: étude iconographique (Corpus vitrearum, France, Etudes II), Paris, 1993, spec. la didascalia e lo schema grafico della fig. 49.

${ }^{28}$ Cfr. A. WATSON, The Early Iconography... op. cit., pp. 151-162 (tabella riassuntiva pubblicata in appendice). 
${ }^{29}$ A. MOMIGLIANO, Dalla Sibilla pagana alla Sibilla cristiana: profezia come storia della religione, in Annali della Scuola Normale Superiore di Pisa, Classe di Lettere e Filosofia, $3^{\text {a }}$ serie, 17, 2, 1987, pp. 407-428.

${ }^{30}$ S. PIAZZA, Genesi e fortuna... op. cit., pp. 820-821, fig. 5.

${ }^{31}$ A. WATSON, The Early Iconography... op. cit., pp. 105-106, tav. XX; R. GAMESON, A scribe's confession and the making of the Anchin Hrabanus (Douai, Bibliothèque Municipale, ms. 340), in Manuscripts in transition : recycling manuscripts, texts and images, B. DEKEYZER, J. VAN DER STOCK (a cura di), Paris, 2005, pp. 65-79.

${ }^{32}$ F. DEUCHLER, Der Ingeborgpsalter, Berlin, 1967, pp. 32-34, tav. X, fig. 18.

${ }^{33}$ N. BROCCA, La tradizione della Sibilla Tiburtina e l'acrostico della Sibilla Eritrea tra oriente e occidente, tardantichità e medioevo : una 'collezione' profetica?, in Antiquité tardive dans les collections médiévales : textes et représentations, VI ${ }^{e}$-XIVe siècle, B. GRÉVIN, S. GIOANNI (a cura di) (Collection de l'École française de Rome, 405) Roma, 2008, pp. 225-260, spec. pp. 228-234.

${ }^{34}$ Ibidem, p. 229.

${ }^{35}$ Agostino, De Civitate Dei, PL, XIII, 23, coll. 281-290. Cfr. F. DE’ MAFFEI, La Sibilla “Tiburtina” e "Prophitissa” nel ciclo degli affreschi di Sant'Angelo in Formis, in Monastica, IV : Scritti raccolti in memoria del XV centenario della nascita di S. Benedetto (480-1980) (Miscellanea cassinese, 48), Montecassino, 1984, pp. 9-30, spec. p. 16 e n. 30; N. BROCCA, La tradizione della Sibilla... op. cit., pp. 228-233.

${ }^{36}$ Agostino, De Civitate Dei, XVIII, 23, 1 (PL, XLI, 1845, col. 579).

${ }^{37}$ E coelo Rex adveniet per saecla futurus: Ibidem.

${ }^{38}$ K. HOFFMANN, The year 1200. A centennial Exhibition at the Metropolitan Museum of Art, NewYork, 1970, vol. I, pp. $267-269$ (scheda n $265:$ "Nativity: St. Matthew”). L'immagine di Cleveland è il recto di un foglio staccato del ms. 142 del Tesoro del Duomo di Treviri: cfr. A. COHEN MUSHLIN, Scriptoria in Medieval Saxony: St. Pancras in Hamersleben, Wiesbaden, 2004, p. 118, nota 43.

${ }^{39}$ Iudicii signum tellus sudore madescet: Agostino, De Civitate Dei, XVIII, 23, 1 (PL, XLI, 1845, col. 579); F. DE’ MAFFEI, La Sibilla... op. cit., pp. $10,15-16$. ${ }^{40} \mathrm{C}$. KOHLER, Un rituel et un bréviaire du Saint-Sépulcre de Jérusalem (XII'-XIII siècle), in Revue de l'Orient latin, 8, 1900-1901, pp. 384-499; B. BAGATTI, Gli antichi edifici... op. cit., p. 66.

${ }^{41}$ C. KOHLER, Un rituel... op. cit., p. 475.

${ }^{42}$ Ibidem.

${ }^{43}$ Ibidem.

${ }^{44}$ J. FOLDA, The Art... op. cit., pp. 357, 360; M. ANDALORO, Da Bisanzio... op. cit., p. 204.

${ }^{45}$ Supra, p. 1.

${ }^{46}$ F. QUARESMIUS, Historica... op. cit., vol. II, p. 645.

${ }^{47}$ V. MILANOVIĆ, The Tree of Jesse... op. cit., p. 50, fig. 4.

${ }^{48} \mathrm{Si}$ vedano, ad esempio, i profeti alla base della cupola della chiesa del monastero di Daphni (fine XI secolo): G. MILLET, Le monastère de Daphni : histoire, architecture, mosaïques, Paris, 1899, tavv. VII-IX.

${ }^{49}$ E. KITZINGER, I mosaici del periodo normanno in Sicilia (III, Il duomo di Monreale : i mosaici dell'abside, della solea e delle cappelle laterali), Palermo, 1994, pp. 34-36, 73-74, figg. 108-127.

${ }^{50}$ B. BERTOLI (a cura di), I mosaici di San Marco. Iconografia dell'Antico e del Nuovo Testamento, Milano, 1988, p. 159, figg. 75c-f, pp. 160-161.

${ }^{51}$ Nell'illustrazione 10b il disegno dell'Albero di Iesse, opera dell'autore, è stato inserito all'interno di un'illustrazione tratta da J. N. SEPP, Jerusalem und das Heilige Land Pilgerbuch nach Palaestina, Syrien und Aegypten, Regensburg, 1875, I, p. 551, ampiamente modificata e adattata allo scopo.

${ }^{52}$ E. KITZINGER, I mosaici... (III), op. cit., pp. 24-25, 71, figg. 1, 11-19.

${ }^{53}$ Idem, I mosaici del periodo normanno in Sicilia (IV, Il duomo di Monreale : i mosaici del transetto), Palermo, 1995.

${ }^{54}$ L. RÉAU, Iconographie de l'art chrétien, II, 2, Paris, 1957, p. 139.

${ }^{55}$ B. BERTOLI (a cura di), I mosaici... op. cit., pp. 44-45, 164.

${ }^{56}$ Ibidem, p. 164.

${ }^{57}$ O. DEMUS, The mosaics of San Marco in Venice, Chicago, 1984, I, 1, p. 9.

58 "Quello marciano [...] rappresenta uno degli interventi cinquecenteschi in basilica più impressionanti per la maniera in cui un tema antico viene rielaborato in chiave moderna, non solo trasportando su di un piano di gigantografia un soggetto diffuso spesso nelle miniature, superando in questo gli esempi delle grandi finestrate o delle sculture nei portali due-trecenteschi, ma anche per la costruzione delle singole figure e per la ricerca di effetti cromatici audaci, quasi dissonanti...": S. MASON, 'Pitture marmoree' della maniera moderna in San Marco, in Arte veneta, 48, 1996, pp. 26-41, spec. pp. 32-33.

${ }^{59}$ S. PIAZZA, Genesi e fortuna... op. cit., pp. 818, 822-823.

\section{JIŠAJEVO STABLO U 12. ST. IZMEĐU ZAPADA I ISTOKA: BILJEŠKE O IZGUBLJENOM MOZAIKU BAZILIKE ROĐENJA ISUSOVA U BETLEHEMU}

Tijekom prvih desetljeća XII. stoljeća na Zapadu počinje kružiti nova ikonografska tema koja ubrzo postiže zavidan uspjeh: Jišajevo drvo kao prikaz biblijske genealogije Krista inspirirane proročanstvom iz Knjige proroka Izaije
(“Isklijat će mladica iz panja Jišajeva, izdanak će izbit' iz njegova korijena. Na njemu će duh Jahvin počivat', duh mudrosti i umnosti, duh savjeta i jakosti, duh znanja i straha Gospodnjeg." Iz 11, 1-2) te u incipit Evanđelja po Mateju 
("Jišaju se rodi David kralj, kralju Davidu rodi se Salomon (...) Jakovu se rodi Josip, muž Marijin, od koje se rodio Isus koji se zove Krist." Mt 1, 1-17).

Prikaz stabla predaka brzo se širi diljem latinskog područja kako nam pokazuju različiti primjeri pronađenih djela u Engleskoj, Francuskoj, Italiji i Španjolskoj. Slično datirani primjeri različitih su dimenzija te su izvedeni na različitim podlogama. Jedan od ranijih monumentalnih primjera, ako ne i najraniji, jest vitraj iz Saint Denisa, koji je naručio opat Suger oko 1140.-1144.

U kratkom će vremenu Jišajevo stablo prijeći granice Europe te doseći do Svete Zemlje: o tome nam svjedoči izvorno postojanje teme među mozaicima bazilike Rođenja Isusova u Betlehemu, dovršene 1169. za vrijeme franačkog kralja Amalrika, 5. kralja Jeruzalema (1162. - 1174.) i bizantskog kralja Emanuela Komnena (1143.-1180.) te Radolfa, betlehemskog biskupa (1155.-1174.). Mozaik je izradio majstor mozaika Efrem, sirijskog podrijetla sudeći po imenu, uz sudjelovanje Venecijanca Zana, čije je ime dijalektalna izvedenica Johannesa, te Grka Bazilija, sudeći prema različitim natpisima koji su dio mozaika.

Prikaz Stabla iz bazilike Rođenja Isusova nije sačuvan, ali nalazimo zapise o njegovom položaju na unutarnjoj strani pročeljnog zida u srednjovjekovnim i ranonovovjekovnim izvorima: u "Libro d'Oltramare" (1345.-1350.) Niccolòa di
Poggibonsija, traktatu o Svetoj Zemlji (1639.) Francesca Quaresmija te u Ciampinijevoj De sacris aedificiis (1693.).

Polazeći od ovih svjedočanstava, članak doprinosi tumačenu dva važna pitanja: važnosti izbora smještaja na ulaznom zidu kao spomen Kristovog rođenja; vjerojatnost zapadnog ikonografskog porijekla zbog manjka prikaza ranijih od 13. stoljeća na istoku Mediterana; formalni izgled prikaza, vjerojatno bizantskog porijekla, uz stilski ductus betlehemskih mozaika jasnog komnenskog izraza; susret Sibile s prorocima u prikazu Stabla kao figure koja nije uvijek bila prikazivana, ali se pojavljuje u latinskom ritusu upravo u kultu Svetog Groba u božićnoj noći; uloge koju je betlehemsko drvo putem širećih utjecaja moglo preuzeti uvodeći figurativnu temu u grčko-istočni kulturni krug te naposljetku moguća razmjena modela s Venecijom zaslugama venetskog majstora Zana koji je radio na mozaiku u bazilici Rođenja, i to ne samo zbog postojanja renesansnog prikaza iste teme u interijeru sv. Marka, možda izvedenog kao zamjena za srednjovjekovnu verziju. Nakon dovršenog istraživanja grafičke rekonstrukcije betlehemskog stabla pružen je uvid u predmet i dana predodžba o istaknutom mjestu koje je stablu dodijeljeno u kompoziciji mozaika.

Preveo: Ivor Kranjec 